\title{
EFFECTS OF GRADUAL ONSET +GZ ON HEMODYNAMIC PARAMETERS AND BRAIN OXYGENATION IN MILITARY PILOTS: PRELIMINARY STUDY
}

\author{
Krzysztof KOWALCZUK', Liana PUCHALSKA ${ }^{3}$, Aleksander SOBOTNICKI², Marek CZERW², \\ Michał JANEWICZ', Mariusz WYLEŻOt', Stefan P. GAŹDZIŃSKI' \\ ${ }^{1}$ Military Institute of Aviation Medicine, Warsaw, Poland \\ 2 Institute of Medical Technology and Equipment ITAM, Zabrze, Poland \\ ${ }^{3}$ Medical University of Warsaw, Poland
}

Source of support: Source of support: The study was supported by the Inspectorate of Military Health Service (01/ WNiL/2007).

Author's address: K. Kowalczuk, Institute of Aviation Medicine, Krasińskiego 54/56 Street, 01-755 Warsaw, Poland, e-mail: kkowalczuk@wiml.waw.pl

Introduction: Sustained acceleration in $+\mathrm{Gz}$ axis may lead to blood mass-volume displacement. Currently, during centrifuge training, this displacement is not assessed directly, but the focus is on its functional correlates, such as the narrowing of the visual field and a decrease in blood oxygenation in the pilot's brain. These are very crude measures that say little about the physiological processes taking place in the pilot's body. Thus, the aim was to evaluate more detailed measures of the cardiovascular system: stroke volume (SV) and cardiac output (CO) and their changes with gradual onset $+\mathrm{Gz}$, as well as changes in frontal brain oxygenation (OX) in relation to $\mathrm{Gz}$, SV, and CO.

Methods: Eight military pilots (six active with different amounts of flight experience) performed the gradual onset rate profile of $\mathrm{Gz}$, Delta $\mathrm{Gz}=0.1 \mathrm{G} / \mathrm{s}$, till $6 \mathrm{G}$. Their SV, CO were evaluated with bioimpedance cardiography, while their OX with near infrared spectroscopy. ECG was constantly monitored.

Results: Increase in Gz led to linear decreases in SV, while CO remained statistically unchanged; however, in most cases, OX decreased linearly with increasing $\mathrm{Gz}$.

Discussion: Linear increase in $+\mathrm{Gz}$ load on human centrifuge results in decreases in cardiac output. Increased heart rate compensates for changes in stroke volume. Nonetheless, brain oxygenation decreases with $\mathrm{Gz}$, likely due to decreased lung gas exchange capacity in hypergravity. Thus, measuring blood oxygenation at the level of the brain may be a better method of monitoring pilots during centrifuge training than bioimpedance cardiography.

Keywords: hemodynamics, cardiac output, stroke volume, head-foot acceleration, $+\mathrm{Gz}$, monitoring, bioimpedance cardiography

Figures: 5 - References: 25 - Full-text PDF: http://www.pjambp.com • Copyright (C 2016 Polish Aviation Medicine Society, ul. Krasińskiego 54/56, 01-755 Warsaw, license WIML • Indexation: Index Copernicus, Polish Ministry of Science and Higher Education 


\section{INTRODUCTION}

Assessment of compensational reflexes during training in high-sustained $G$ is of great diagnostic importance. Currently, ECG (and ECG-derived heart rate), blood saturation and blood pulsation at the earlobe, as well as the narrowing field of vision are used to assess the blood mass-volume displacement under acceleration in $+G z$ axis (head-foot direction). The narrowing of the visual field is a sign of decrement in retinal blood flow and decreasing blood saturation may precede the onset of G-LOC (G -force induced loss of consciousness). It has been demonstrated that brain oxygen saturation may be monitored in flight [9] during AGSM (Anti-G straining Manoeuvre) training $[4,8,19]$ and that the drop in oxygen saturation precedes loss of consciousness. However, the drop in blood oxygenation preceded G-LOC by only a few seconds [19].

Impedance cardiography (ICG) is a noninvasive technology measuring total electrical conductivity of the thorax and its changes in time to process continuously a number of cardiodynamic parameters, such as Stroke Volume, SV, Heart Rate, HR, Cardiac Output, CO, Ventricular Ejection Time (VET), etc. It is used to detect the impedance changes caused by a high-frequency, low-value current flowing through the thorax or another organ such as the neck or limb. ICG is also known as electrical impedance plethysmography (EIP) or Thoracic Electrical Bioimpedance (TEB).

ICG was extensively validated against other established methods. Electrical bioimpedance methods were often used to monitor cardiac output (CO) and stroke volume (SV) [14] and were validated against other methods, such as Doppler ultrasound (Echo Doppler) [2,5,21], multigated radionuclide cardiography [6]. Additionally, in animal models, ICG was validated against thermodilution and Doppler ultrasound across a wide range of blood flow parameters [5], as well as against stroke volumes obtained with dye dilution and electromagnetic flowmeters in dogs [15]. Finally, ICG methods were used to demonstrate vascular vasoconstriction in humans during exercise [12]. In humans, electrical bioimpedance methods were used to non-invasively and simultaneously measure cardiac and peripheral (limb) blood flow [21].

Given the proven reliability of the ICG method, we evaluated its potential use to monitor blood mass-volume movements in military pilots while under sustained accelerations in $\mathrm{Gz}$ axis. Here, we focus on the Gradual Onset Rate (GOR) profile that is generally used in aviation medicine diagnostics.

\section{METHODS}

\section{Subjects}

Eight male military pilots (age: $34.8 \pm 8.2$ years; 24-47 years; five in active duty; two working mostly in the office, and one retired, currently piloting civilian aircraft), with different amounts of flight experience, participated in the study. All subjects had current fitness to fly certificates issued by the Aeromedical Board (i.e. they were healthy). They all had normal or corrected-to-normal vision. The study protocol was approved in advance by the Bioethical Committee of the Military Institute of Aviation Medicine in Warsaw. Each subject provided written informed consent before participating and they were paid for taking part in the experiment.

\section{Equipment}

A human centrifuge HTCO7 (AMST, Braunau, Austria) was used to produce Gz. It is a flight simulator giving opportunities for using it in operational, training and diagnostic profiles. It permits intensive pilot training providing enhanced performance of anti-G maneuvers and familiarization of aircrews with the effects of high accelerations and push-pull phenomena. Moreover, the centrifuge provides a safe alternative to raise awareness of aircrews in the case of occurrences of unwanted effects of accelerations, such as G-LOC loss of consciousness or spatial disorientation. The gondola of the centrifuge is assembled on an 8-meter-long arm and allows to achieve Z-axis accelerations in the range from $-3 \mathrm{Gz}$ to $+16 \mathrm{Gz}$ with the maximal onset of accelerations over $14.5 \mathrm{G} / \mathrm{s}$. Additionally, gyroscopic suspension of the gondola allows to achieve $\mathrm{X}$-axis and $\mathrm{Y}$-axis accelerations respectively in the range of the values $\pm 10 \mathrm{G}$ and $\pm 6 \mathrm{G}$. Interchangeable parts of the centrifuge gondola facilitate functional projection cockpit equipment of the Polish Air Forces basic multi-purpose aircraft i.e. F-16 Block 52+ and MiG-29. The centrifuge is equipped with multiple devices to monitor the physiological effects elicited by the accelerations on the pilot. Of the available measures, we recorded ECG, heart rate, and Gz value. Mean blood oxygenation (both oxygenated and de-oxygenated blood) of the frontal lobe of the brain (OX) was measured using near infrared spectroscopy (NIRS; NelcorTM Pulse Oximetry, Covidien-Medtronic, Dublin, Ireland). OX was averaged in $4 \mathrm{~s}$ intervals. The optode was attached to the forehead of the pilot.

Stroke volume (SV) and cardiac output (CO) were measured using electrical bioimpedance. A 3-channel experimental module ReoWir (ITAM, 
Zabrze, Poland) was used to measure electrical bioimpedance simultaneously in the thorax and in the neck. The module allows to register the signal component of variable impedance $\Delta Z$ or its first derivative $\mathrm{dZ} / \mathrm{dt}$ and measure the base impedance $\mathrm{ZO}$ in three channels. The measurement of electrical impedance is implemented using the constant current tetrapolar method. A high frequency $(40 \mathrm{kHz})$ of the current of a small constant amplitude $\left(1 \mathrm{~mA}_{\mathrm{RMS}}\right)$ flowing through the body results in a voltage drop across the impedance of the tissues. This voltage signal is amplified, demodulated, and digitized with 24-bit resolution. The firmware im-

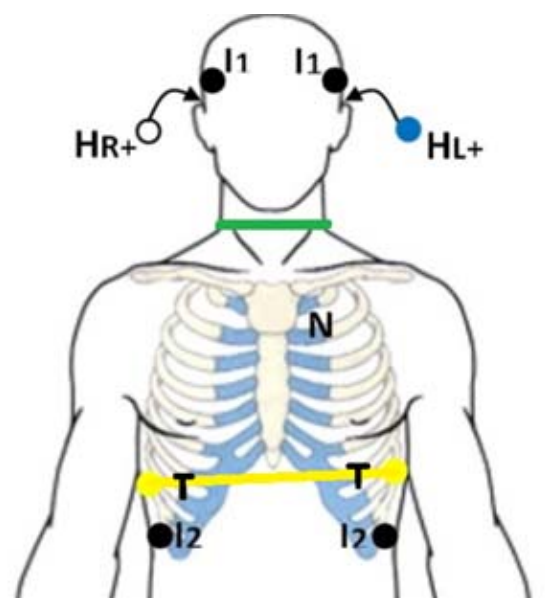

Fig. 1. Location of electrodes: Current electrodes: I1 placed on temples (gel electrodes), 12 placed on the thorax (spring electrode), receiver electrodes $\mathrm{HI}+$ and $\mathrm{HR}+$ - behind the ears (gel electrode), $\mathrm{N}$ placed on the bottom of the neck (spring electrode). plements the separation of base impedance $\mathbf{Z 0}$ from the variables component $\Delta \mathrm{Z}$.

ReoWir module meets the safety requirements of European standard EN 60601-1 for medical equipment confirmed by a report from an accredited laboratory. Metrological parameters of the module were verified by using a dedicated simulator of resistive parameters of the tissues - ReoTester [16].

The location of electrodes on the pilot is depicted in Fig. 1. Bioimpedance signal from the thorax is collected using a standard electrode arrangement, as in the Kubicek method [6], but the location of the electrodes on the head is an original arrangement. Both NIRS and ReoWir were connected to the system of the centrifuge, thus OX and bioimpedance signals were recorded synchronously with ECG, heart rate, Gz and saved in the centrifuge's system. Then, all data were anonymized and exported for further processing on external computers.

\section{Procedure}

The subjects were briefed on the study and its aims. Then ECG and bioimpedance leads were connected, and the NIRS optode was attached to the forehead. The Gradual Onset Rate (GOR) profile was selected, as it is the most common profile used in aviation medicine diagnostics. It is characterized by a linear increase in $\mathrm{Gz}$ at $0.1 \mathrm{G} / \mathrm{s}$ rate, until $6 \mathrm{G}$ is reached. Then the centrifuge is decelerated at $-0.5 \mathrm{G} / \mathrm{s}$ (see Fig. 2)

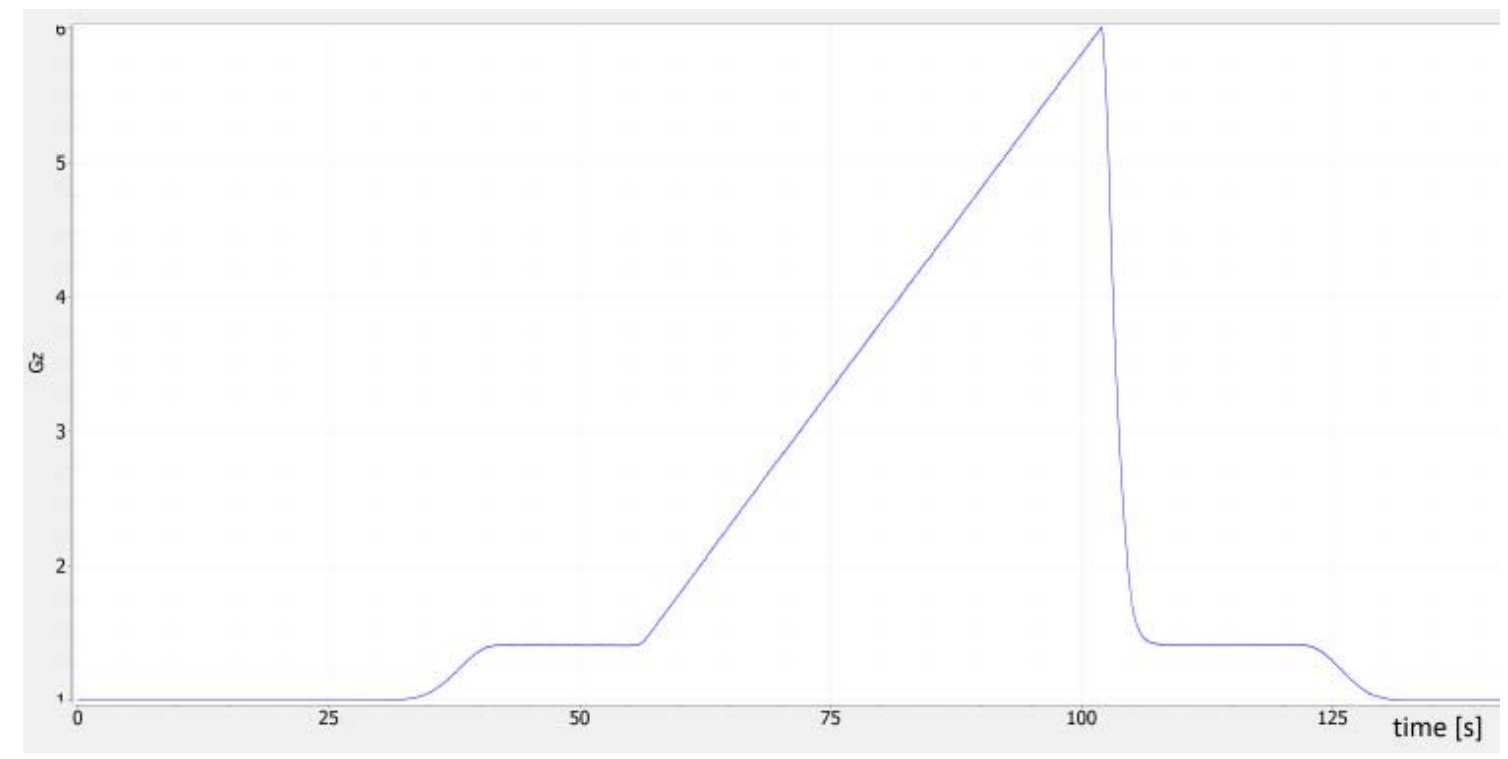

Fig. 2. Gz acceleration as a function of time in the GOR profile. After initial centrifuge startup procedure (increase up to $1.4 \mathrm{Gz}), \mathrm{Gz}$ increased linearly with time, until $+6 \mathrm{Gz}$ was reached. 


\section{Calculation of stroke volume}

Stroke volume (SV) of the heart was calculated based on the Kubicek formula [20],

$$
S V=\text { constant }\left(\frac{1}{Z_{0}}\right)^{2}\left[\operatorname{LVET}\left(\frac{d_{z}}{d_{t}}\right)_{\max }\right]
$$

where $Z_{0}$ is the base impedance measured directly during the experiment. $Z_{0}$ values measured at the beginning of LVET were taken for calculations. Left-ventricular ejection time (LVET) and (dZ/dt) max were traced manually by two independent raters, trained and supervised by LP, who has extensive expertise in bioimpedance methods. The method is depicted in Fig.3. In short, onsets and ends of the ejection phase were marked manually by the raters, thus the left ventricle ejection times (LVET) were established. Within these intervals, respective maxima and minima were automatically detected by our purpose-written software, thus (dZ/dt)max were established. Identified artifacts were excluded from analyses. SV and CO were calculated.

For the neck, only (dZ/dt)max was marked; it can be interpreted as the flow index in the carotid arteries $[7,22]$.

\section{Statistical Analysis}

Percentage changes of all measures of interest were scaled by means of average respective values at $1 \mathrm{G}$. The normality of distributions was evaluated automatically with appropriate tests. Linear regressions were used to evaluate relationships between normalized values of SV, CO, brain blood oxygenation, heart rate, and $\mathrm{Gz}$, for indi- vidual pilots. We acknowledge that the bioimpedance signals were affected by artifacts created by the subject's breathing; however, these artifacts were statistically 'averaged out' by the regression procedure, similarly to signal averaging applied in commercially available equipment. Additionally, relationships between normalized values of SV, CO and blood oxygenation were evaluated using linear regression. All tests were performed using Statistica 13.1 software (Dell, Round Rock, TX, USA).

\section{RESULTS}

Analyses of individual data demonstrated nonlinearity in the relationships between NIRS, heart rate, and $\mathrm{Gz}$.

For thorax, the base impedances, compared to respective impedances at $1 \mathrm{G}$, were lower of the order of $0.4 \pm 0.5 \Omega$ at low accelerations (range $0 \div-1.8 \Omega$ ), while they generally increased on average by $0.35 \pm 0.64 \Omega$ at higher accelerations (range $-0.8 \div+1.2 \Omega$ ), likely reflecting blood mass displacements out of the thorax due to hypergravity (AGSM; anti-gravity straining manoeuvres). However, for the neck and compared to respective impedances at $1 \mathrm{G}$, the impedances increased on average by $0.64 \pm 0.69 \Omega$ and $0.47 \pm 0.90 \Omega$ for low and high G-loads, respectively. The respective ranges were $(-0.41 \div-2.04 \Omega)$ and $(-1.22 \div+1.63 \Omega)$.

The slopes in linear regressions of the individual relationships between SV and Gz were generally significantly negative, attesting that SV decreases with increasing Gz. Fig. 4 depicts exemplary data. CO demonstrated a different relationship: in the

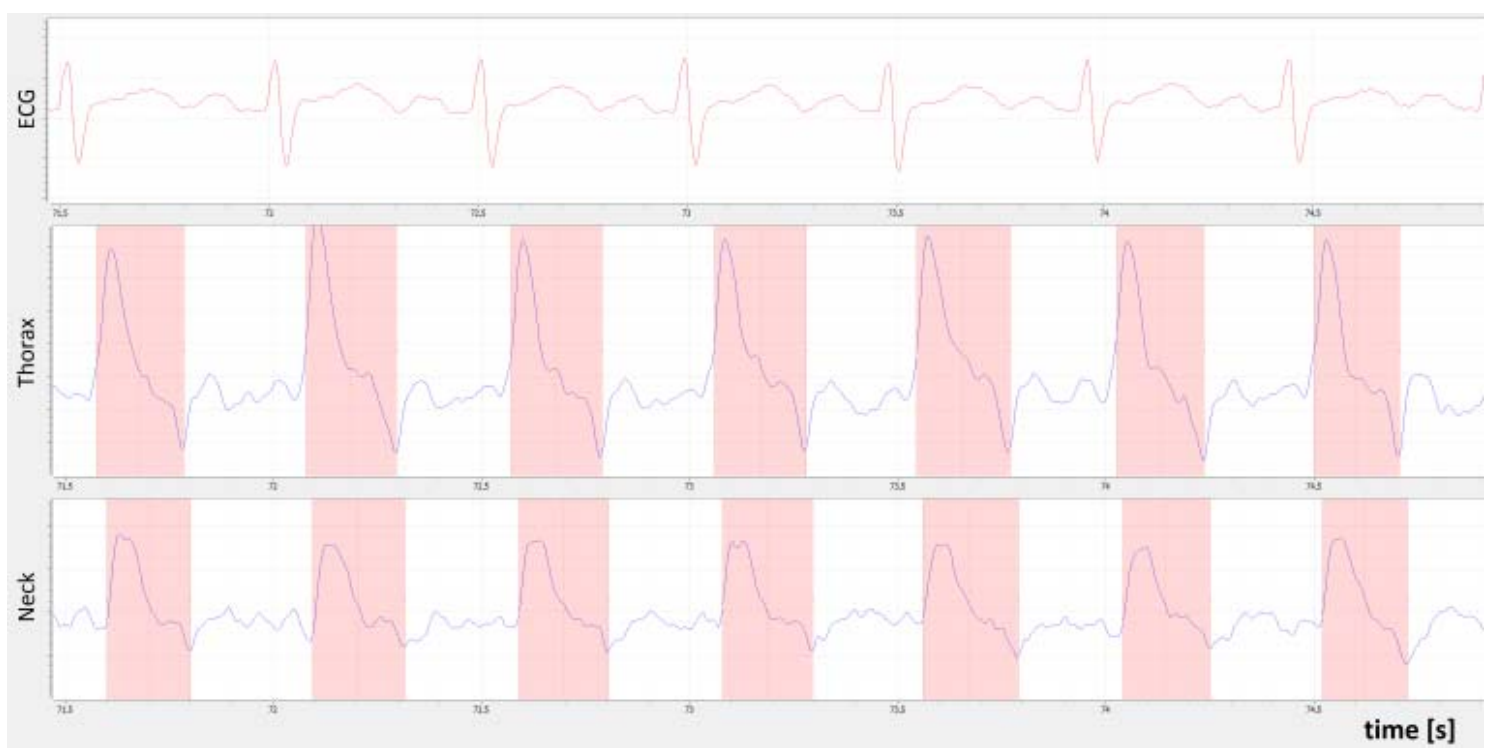

Fig. 3. ECG signal and derivatives of thorax and neck bioimpedance signals. The red areas depict the left ventricle ejection time (LVET). 
majority of pilots, an initial increase in $\mathrm{CO}$ was observed, which was followed by a decrease in $\mathrm{CO}$ at higher $\mathrm{Gz}$ accelerations. However, in two participants, the CO decreased linearly with $\mathrm{Gz}$, and in another two it increased. Nonetheless, OX generally linearly decreased with increasing $\mathrm{Gz}$ load, as exemplified in Fig. 5.

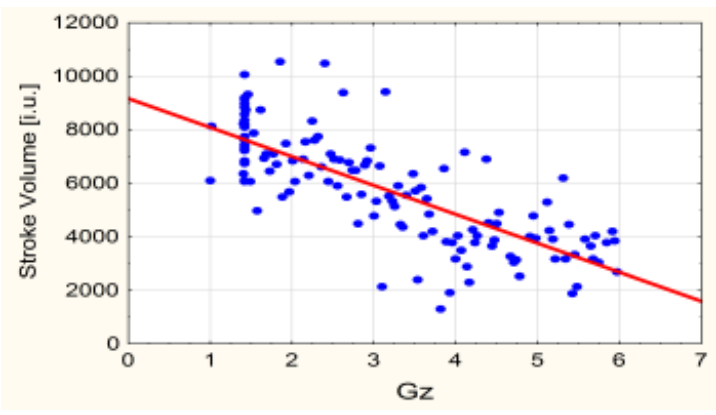

Fig. 4. Changes in stroke volume (SV) as a function of $\mathrm{Gz}$ (aggregated data from all pilots).

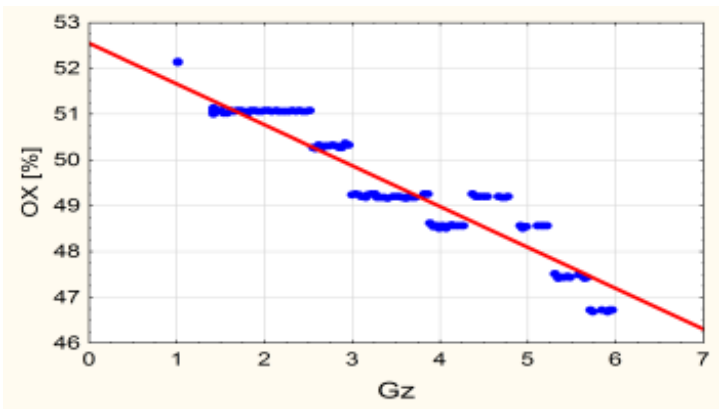

Fig. 5. Brain blood oxygenation at prefrontal lobe as a function of $\mathrm{Gz}$ (representative figure).

We did not find any significant correlations between flow index in the carotid arteries and Gz, SV, or CO. OX was generally significantly related to CV (mean slope: $+0.024 \pm 0.029$ ) but not to CO (mean slope: $+0.003 \pm 0.029$ ).

\section{DISCUSSION}

In this study, we demonstrated that linear increase in $+\mathrm{Gz}$ acceleration led to linear decreases in stroke volume. These changes were compensated by increased heart rate, which resulted in unchanged cardiac output in the range up to $6 \mathrm{G}$. However, larger $G$ loads resulted in statistically significant decreases of brain frontal lobe oxygenation, most likely due to the redistribution of the blood in the thorax; i.e. less blood ended up in the brain due to the larger gravitational field in the centrifuge. An additional $\mathrm{Gz}$ effect that must be taken into consideration is air-blood mismatch in lungs under $\mathrm{Gz}[3,11,24]$.
All examined pilots were neither instructed to perform or withdraw from performing AGSM. However, they performed a muscular component of AGSM without breathing component. The breathing component of AGSM relies on the intermittent increase of airway pressure which can at least partially alleviate Gz effects in the lungs. Surprisingly, brain oxygenation was more strongly related to cardiac volume than to cardiac output.

Our results are not in line with a previous study by Rohdin et al. [17], who reported 30-40\% smaller $\mathrm{CO}$ in healthy volunteers at $5 \mathrm{G}$, compared with 1G. We invited military pilots, who are a selected population and trained to resist the effects of hypergravity. They perform muscular tensioning under $\mathrm{Gz}$ as a habit emerging from flight experience. Thus, the differences in the results likely reflect the two different cohorts participating in both studies. This conclusion is further supported by several studies $[1,13,23]$, which did not find significant changes in CA of young participants at $+\mathrm{Gz}$ below 2G. However, they reported decreases in SV at those accelerations, which is consistent with our results.

Rohdin et al. [17] also demonstrated decreased lung diffusion capacity in hypergravity. On the other hand, increases in base impedance of the neck with increasing $G z$, could be interpreted as a decrease of the blood volume in the neck. However, cranium acts as a rigid container of the brain, cerebro-spinal fluid, and blood, which are virtually incompressible [18], thus the amount of blood in the brain is likely constant during $+\mathrm{Gz}$ accelerations used in our study. Moreover, cerebral autoregulation maintains relatively constant cerebral blood flow within the range of arterial blood pressure from about 60 to $150 \mathrm{mmHg}[10,18]$. In our experience, arterial blood pressure may decrease below $60 \mathrm{mmHg}$ at $+6 \mathrm{G}$, thus smaller cerebral blood flow may contribute to decreases of OX with increasing $+\mathrm{Gz}$.

The above-mentioned phenomena could be responsible for decreases in OX in our pilots, despite no apparent changes in cardiac output. Given our relationship between brain oxygenation and stroke volume, but not cardiac output, we believe that further research into lung gas exchange capacity in hypergravity is warranted.

We did not evaluate changes in arterial pressure and its relationship to the measured parameters. As it is known that Z0 increases with bleeding induced hypotension in pigs [25], it is likely that changes in blood pressure would explain some variance in $\mathrm{SV}, \mathrm{CO}$, and $\mathrm{OX}$. 
Our results may help understand physiological adaptations to hypergravity in commercial space flights. Although the $\mathrm{G}$ loads in these voyages are not expected to surpass 4G, one should remember that military pilots are selected based on endurance and fitness. Thus, the physiological processes described in our study may take place at much lower accelerations in the general population. The non-zero resistance of the current electrodes applied in our study lead to some limitations in the results, as it caused a slight overestimation of baseline impedances, but, it resulted in slight underestimation of the reported changes in SV and CO. Similarly, the breathing artifacts had detrimental effects on fitting quality; however, it is unlikely it biased the regression results.

The conclusions are limited by the fact that the pilots performed straining at various times of the training; some of them at the very beginning of the profile, once stopping at higher Gz ac- celerations. These maneuvers could have caused variability in the measured relationship between stroke volume, cardiac output, and Gz. Nonetheless, brain oxygenation decreased in all pilots with increasing $\mathrm{Gz}$.

\section{CONCLUSIONS}

In conclusion, a linear increase in $\mathrm{G}$ load on human centrifuge results in decreases in stroke volume. Increased heart rate compensates for changes in cardiac output, although there are limitations to this mechanism. Nonetheless, brain oxygenation decreases with $\mathrm{Gz}$, possibly due to decreased lung diffusion capacity in hypergravity. Thus, measuring blood oxygenation at the level of the brain may be a better method of monitoring pilots during centrifuge training than bioimpedance cardiography.

We thank Mariusz Walas, Jan Lech, Krzysztof Król, and Krzysztof Skórzyński for their technical assistance, Hanna Palonek for the manual marking of the bioimpedance signals, and Andrzej Gaździński for his assistance in computer programming needed for data analyses. Last but not least, we thank W. Franciszek Skibniewski for his support and encouragement along the way.

\section{AUTHORS' DECLARATION:}

Study Design: Krzysztof Kowalczuk, Liana Puchalska, Michał Janewicz, Stefan P. Gaździński; Data Collection: Krzysztof Kowalczuk, Liana Puchalska, Aleksander Sobotnicki, Marek Czerw, Michał Janewicz, Stefan P. Gaździński; Manuscript Preparation: Krzysztof Kowalczuk, Aleksander Sobotnicki, Mariusz Wyleżoł, Stefan P. Gaździński; Funds Collection: Mariusz Wyleżoł. The Authors declare that there is no conflict of interest.

\section{REFERENCES}

1. Arvedsen SK, Eiken O, Kolegard R, Petersen LG, Norsk P, Damgaard M. Body height and arterial pressure in seated and supine young males during $2 \mathrm{G}$ centrifugation. American Journal of Physiology-Regulatory Integrative and Comparative Physiology. 2015;309(9):R1172-R7.

2. Bodo M. Studies in Rheoencephalography (REG). Journal of Electrical Bioimpedance. 2010;1:18-40.

3. Buick F, Hartley J, Pecaric M. Maximum intrathoracic pressure with Anti-G straining maneuvers and positive pressure breathing during +GZ. Aviation Space and Environmental Medicine. 1992;63(8):670-7.

4. Caiozzo VJ, Rose-Gottron C, Baldwin KM, Cooper D, Adams G, Hicks J, et al. Hemodynamic and metabolic responses to hypergravity on a human-powered centrifuge. Aviation Space and Environmental Medicine. 2004;75(2):101-8.

5. Castor G, Klocke RK, Stoll M, Helms J, Niedermark I. Simultaneous measurement of cardiac-output by thermodilution, thoracic electrical bioimpedance and doppler ultrasound. British Journal of Anaesthesia. 1994;72(1):133-8.

6. Gujjar AR, Muralidhar K, Bhandopadhyaya A, Sathyaprabha TN, Janaki P, Mahalla BK, et al. Transthoracic electrical bioimpedence cardiac output: comparison with multigated equillibrium radionuclide cardiography. Journal of Clinical Monitoring and Computing. 2010;24(2):155-9.

7. Khalil SF, Mohktar MS, Ibrahim F. The Theory and Fundamentals of Bioimpedance Analysis in Clinical Status Monitoring and Diagnosis of Diseases. Sensors. 2014;14(6):10895-928. 
8. Kobayashi A, Kikukawa A, Kimura M, Inui T, Miyamoto Y. Cerebral Near-Infrared Spectroscopy to Evaluate Anti-G Straining Maneuvers in Centrifuge Training. Aviation Space and Environmental Medicine. 2012;83(8):790-4.

9. Kobayashi A, Tong A, Kikukawa A. Pilot cerebral oxygen status during air-to-air combat maneuvering. Aviation Space and Environmental Medicine. 2002;73(9):919-24.

10. Koller A, Toth P. Contribution of Flow-Dependent Vasomotor Mechanisms to the Autoregulation of Cerebral Blood Flow. Journal of Vascular Research. 2012;49(5):375-89.

11. Macdougall JD, McKelvie RS, Moroz DE, Moroz JS, Buick F. The effects of variations in the Anti-G straining maneuver on blood-pressure at $+G z$ acceleration. Aviation Space and Environmental Medicine. 1993;64(2):126-31.

12. Morgan AJ, Hosking SL. Non-invasive vascular impedance measures demonstrate ocular vasoconstriction during isometric exercise. British Journal of Ophthalmology. 2007;91(3):385-90.

13. Ogawa Y, Yanagida R, Ueda K, Aoki K, Iwasaki K-i. The relationship between widespread changes in gravity and cerebral blood flow. Environmental Health and Preventive Medicine. 2016;21(4):186-92.

14. Onozawa A, Kikukawa A, Miyamoto $\mathrm{Y}$. A new evaluation method for $+\mathrm{Gz}$ tolerance with loratadine by using a near-infrared spectroscopy. Dynamic medicine : DM. 2008;7:3-.

15. Patterson RP, Witsoe DA, From A. Impedance stroke volume compared with dye and electromagnetic flowmeter values during drug-induced inotropic and vascular changes in dogs. In: Riu PJ, Rosell J, Bragos R, Casas O, editors. Electrical Bioimpedance Methods: Applications to Medicine and Biotechnology. Annals of the New York Academy of Sciences. 8731999. p. 143-8.

16. Piekar E, Sobotnicki A. The simulator of resistive parameters of the tissues for bioimpedance research. Measurement Automation and Monitoring. 2014;60:1181-4.

17. Rohdin M, Petersson J, Sundblad P, Mure M, Glenny RW, Lindahl SGE, et al. Effects of gravity on lung diffusing capacity and cardiac output in prone and supine humans. Journal of Applied Physiology. 2003;95(1):3-10.

18. Rudzinski W, Swiat M, Tomaszewski M, Krejza J. Cerebral hemodynamics and investigations of cerebral blood flow regulation. Nuclear medicine review Central \& Eastern Europe. 2007;10(1):29-42.

19. Ryoo HC, Sun HH, Shender BS, Hrebien L. Consciousness monitoring using near-infrared spectroscopy (NIRS) during high plus Gz exposures. Medical Engineering \& Physics. 2004;26(9):745-53.

20. Sherwood A, Allen MT, Fahrenberg J, Kelsey RM, Lovallo WR, Vandoornen LP. Methodological guidelines for impedance cardiography. Psychophysiology. 1990;27(1):1-23.

21. Stanley AWH, Jr., Herald JW, Athanasuleas CL, Jacob SC, Bartolucci AA, Tsoglin AN. Multi-channel electrical bioimpedance: a non-invasive method to simultaneously measure cardiac output and individual arterial limb flow in patients with cardiovascular disease. Journal of Clinical Monitoring and Computing. 2009;23(4):243-51.

22. Stanley AWH, Jr., Herald JW, Athanasuleas CL, Jacob SC, Sims SW, Bartolucci AA, et al. Multi-channel electrical bioimpedance: a new noninvasive method to simultaneously measure cardiac and peripheral blood flow. Journal of clinical monitoring and computing. 2007;21(6):345-51.

23. Ueda K, Ogawa Y, Yanagida R, Aoki K, Iwasaki K-i. Dose-Effect Relationship Between Mild Levels of Hypergravity and Autonomic Circulatory Regulation. Aerospace Medicine and Human Performance. 2015;86(6):535-40.

24. Whinnery JE. +GZ - tolerance correlation with clinical-parameters. Aviation Space and Environmental Medicine. 1979;50(7):736-41.

25. Venugopal D, Patterson R, Jhanjee R, McKnite S, Lurie KG, Belalcazar A, et al. Subcutaneous Bioimpedance Recording: Assessment of a Method for Hemodynamic Monitoring by Implanted Devices. Journal of Cardiovascular Electrophysiology. 2009;20(1):76-81.

\section{ACKNOWLEDGEMENTS}

The views, opinions, and findings contained in this article are our own and should not be construed as an official Polish Air Force position, policy, or decision, unless so designated by other official documentation.

Cite this article as: Kowalczuk K, Puchalska L, Sobotnicki A, Czerw M, Janewicz M, Wyleżoł M, Gaździński SP. Effects of Gradual Onset $+\mathrm{Gz}$ On Hemodynamic Parameters And Brain Oxygenation in Military Pilots: Preliminary Study. Pol J Aviat Med Bioeng Psychol 2016; 22(3): 5-11. DOl: 10.13174/ pj ambp. 12.07.2017.01 